\title{
Global Characterization of the CEC 2005 Fitness Landscapes Using Fitness-Distance Analysis
}

\author{
Christian L. Müller and Ivo F. Sbalzarini \\ Institute of Theoretical Computer Science and Swiss Institute of Bioinformatics, \\ ETH Zurich, CH-8092 Zurich, Switzerland \\ christian.mueller@inf.ethz.ch, ivos@ethz.ch \\ http://www.mosaic.ethz.ch
}

\begin{abstract}
We interpret real-valued black-box optimization problems over continuous domains as black-box landscapes. The performance of a given optimization heuristic on a given problem largely depends on the characteristics of the corresponding landscape. Designing statistical measures that can be used to classify landscapes and quantify their topographical properties is hence of great importance. We transfer the concept of fitness-distance analysis from theoretical biology and discrete combinatorial optimization to continuous optimization and assess its potential to characterize black-box landscapes. Using the CEC 2005 benchmark functions, we empirically test the robustness and accuracy of the resulting landscape characterization and illustrate the limitations of fitness-distance analysis. This provides a first step toward a classification of real-valued black-box landscapes over continuous domains.
\end{abstract}

Keywords: Fitness landscape, landscape characterization, fitness-distance correlation, continuous black-box optimization.

\section{Introduction}

Real-valued optimization problems over continuous parameter spaces ("continuous optimization problems") are ubiquitous in science and engineering. They occur in many practical applications ranging from simple parameter identification in data-model fitting to intrinsic design-parameter optimization in complex technical systems. In a blackbox optimization problem only zeroth-order information about the objective function is available. The objective function may be discontinuous or noisy, and analytic gradients or higher-order information may be unknown or inexistent. The diversity of real-world continuous black-box optimization problems hampers a clean classification of problem structure and complexity. Nevertheless, an interesting approach is provided by the landscape metaphor. Ever since Sewall Wright introduced the fitness landscape imagery to evolutionary biology [1] it has been a highly influential concept in many subfields of biology and, more recently, also in combinatorial optimization [2].

We advocate that the fitness landscape perspective also offers a way to establish a more refined analysis of continuous black-box optimization problems. Inspired by our shared visual experience of natural terrains and sceneries, we consider the continuous input variables a high-dimensional landscape domain. Neighborhood or nearness in this landscape domain is defined by a suitable distance metric. We interpret the

C. Di Chio et al. (Eds.): EvoApplications 2011, Part I, LNCS 6624, pp. 294 3032011.

(C) Springer-Verlag Berlin Heidelberg 2011 
scalar objective function value as a height or elevation over the landscape domain. The landscape metaphor encourages a characterization in terms of topographical features, such as valleys, ridges, mountain peaks, and plateaus. In order to underline our view of black-box optimization problems as high-dimensional, complex landscapes we use the term black-box landscape. It is conceivable that certain landscape topologies allow efficient optimization while others almost surely lead to failure of a given search heuristic. Despite the tremendous number of novel continuous black-box optimization heuristics published in the past two decades, limited attention has been paid to the question what global topology a certain problem instance has, how to quantify it, and how success or failure of a certain algorithm can be related to landscape topology. In this paper we attempt a first step toward filling this gap. We propose to characterize real-valued black-box landscapes solely based on zeroth-order information, i.e., within a statistical sampling framework. We transfer the well-known concepts of fitness-distance plots and fitness-distance correlation from evolutionary biology and combinatorial optimization into the continuous black-box optimization context.

This paper is structured as follows: We first present a number of conceivable landscape topologies and comment on their impact on the performance of continuous search heuristics. In Section 3 we consider the concepts of fitness-distance plots and fitnessdistance correlation. After a short review of the topic we present a number of tools that are applicable to continuous black-box landscapes. We apply these techniques to the IEEE CEC 2005 benchmark functions in Section 4 in order to test their capacity to quantify certain landscape topologies. We conclude this work and suggest future studies in Section 5 .

\section{Landscape Topologies and Their Impact on Continuous Black-Box Search Heuristics}

We sketch a number of conceivable landscape topologies in Fig. 1. The simplest topology is a convex structure (Fig. 1a). This landscape has only one minimum, the global one. If one knows in advance that both the landscape domain and the objective function are convex, there is a wealth of exact and efficient techniques for finding this global minimum. A globally convex single-funnel landscape topology (Fig. 1b) consists of a number of local minima that can be seen as high-frequency perturbations to an underlying convex structure. Functions with this topology, also known as "big valley structures" [2], have been analyzed theoretically by Hu and co-workers [3]. In the evolutionary optimization community, Hansen and Kern [4] pointed out that "if the local optima can be interpreted as perturbations of an underlying unimodal function", the Evolution Strategy with Covariance Matrix Adaptation (CMA-ES) performs well. The well-known Rastrigin function and the Ackley function belong to this class of landscapes. This observation led Lunacek and Whitley to introduce the dispersion metric [5] as statistical measure that attempts detecting such landscape topologies and may thus serve as a predictor for success or failure of CMA-ES. Both convex and globally convex landscapes are also termed "single-funnel landscapes" [5]6]. Another archetypal landscape structure is the "double-funnel topology" (Fig. 1k). Whenever the funnel that contains the global minimum covers a much smaller part of the domain than the other funnels, this 

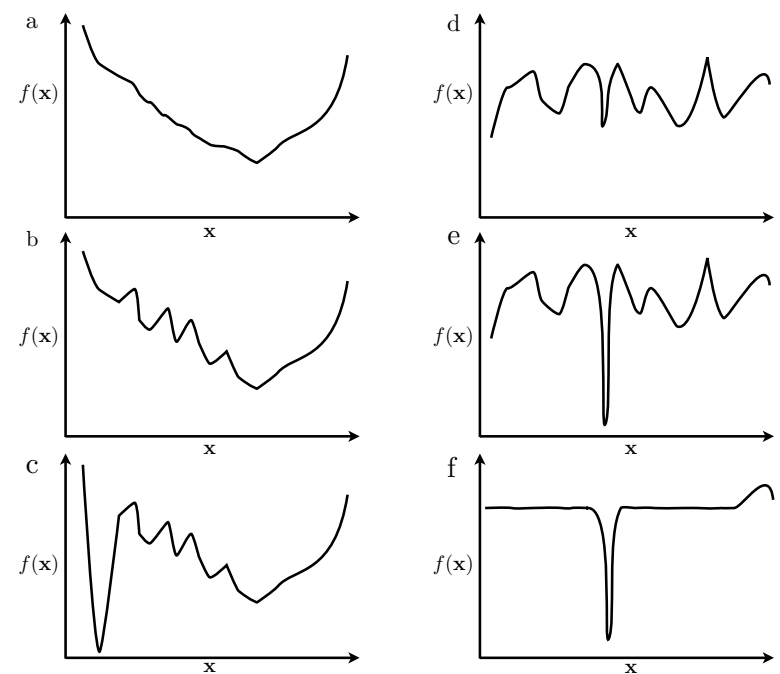

Fig. 1. Sketches of archetypal landscape topologies. (a) Convex single-funnel landscape. (b) Globally convex single-funnel landscape. (c) Double-funnel landscape with a broad sub-optimal funnel. (d) Multi-modal landscape with minimum at the boundary and no funnel structure. (e) Same as (d) but with a deep, needle-like minimum. (f) Golf-course or needle-in-a-haystack topology with large regions of neutrality.

topology poses considerable challenges for search heuristics. Most searches are drawn toward the bottom of the sub-optimal funnel. In the black-box optimization community such a landscape is also called deceiving. Ikeda and Kobayashi called such topologies "UV-structures" and showed the failure of a standard genetic algorithm (GA) on certain model instances. Sakuma and Kobayashi subsequently proposed a novel real-coded GA to overcome the observed performance loss [7]. Lunacek and co-workers also studied the performance of heuristics on double-funnel landscapes in their paper "The impact of global structure on search" [8]. They showed that the performance of CMA-ES can considerably decrease on such instances. The double-funnel case is the simplest instance of the class of multi-funnel landscapes. A real-world, tunable double-funnel benchmark test case based on Lennard-Jones clusters has been designed by Müller and Sbalzarini [9]. Müller and co-workers also introduced a parallel CMA-ES algorithm, the Particle Swarm CMA-ES (PS-CMA-ES) that is able to improve the performance of CMAES on general multi-funnel landscapes [6]. Figures 1 1 d and e show general multimodal landscapes with no global funnel structure. The notoriously hard golf-course or needlein-a-haystack topology, where large flat regions surround a single narrow minimum, is depicted in Fig. 1f.

In most black-box optimization scenarios the topology of the considered problem is not known. Except for Lunacek and Whitley's dispersion metric, general tools and techniques for quantifying or classifying the global topology of a landscape based on only zeroth-order information are largely missing. A potentially useful candidate 
is the concept of fitness-distance analysis from evolutionary biology and combinatorial optimization. In the next section we propose this concept for the global characterization of continuous black-box landscapes.

\section{Fitness-Distance Analysis}

Fitness-distance analysis quantifies the relation between the fitness of a collection of points in the landscape and their distances to the global minimum. It has first been introduced in theoretical biology by Kauffman for the analysis of NK landscapes [10]. Kauffman introduced discrete NK landscapes as a model for the maturation of immune response [11]. Boese used fitness-distance analysis for the symmetric Traveling Salesman Problem (TSP) [2]. He observed a strong fitness-distance correlation (FDC), which led him to formulate the big-valley hypothesis for certain TSP instances. This hypothesis states that certain TSP instances have a globally convex landscape structure, which may explain the success of certain heuristic approaches for solving TSP. Jones and Forrest [12] introduced fitness-distance analysis and the corresponding correlation coefficients as a "measure of problem difficulty" for GA's on combinatorial optimization problems. Ever since, FDC analysis has been applied to many discrete optimization problems, including the Graph Bi-Partitioning problem [13], the Job-shop Scheduling Problem (JSP) [14], and the unconstrained binary Quadratic Assignment problem [15]. For optimization problems over continuous search spaces, however, quantitative studies using fitness-distance analysis are largely missing.

\subsection{Fitness-Distance Analysis for Continuous Black-Box Landscapes}

We propose to characterize the global topology of real-world black-box landscapes using fitness-distance analysis. Wang and Li proposed the fitness-distance correlation independently of us in the context of continuous NK landscapes [16]. We consider black-box landscapes $\mathcal{L}_{\mathrm{B}}$ defined by a triplet $\left(\mathcal{X}, d_{\mathrm{E}}, f\right)$ where the landscape domain $\mathcal{X}$ is box-constrained with $\mathcal{X}=[\mathbf{l}, \mathbf{u}] \subset \mathbb{R}^{n}$. The vectors $\mathbf{l}, \mathbf{u} \in \mathbb{R}^{n}$ define the lower and upper bounds. For unconstrained problems, fitness-distance analysis can be applied to any box-shaped region of interest of the landscape. Distances between points in the landscape domain are measured using the Euclidean distance $d_{\mathrm{E}}$. Given a uniformly random sample $\mathbf{x}^{(j)} \in \mathcal{X}, j=1, \ldots, S$ from the landscape, we evaluate the fitness function $f$ at the sampled points and denote the values by $f^{(j)} \in \mathbb{R}, j=1, \ldots, S$. In standard fitness-distance analysis the location of the global minimum $\mathbf{x}_{\min }$ is assumed to be known a priori. While in a benchmark scenario this information is available, $\mathbf{x}_{\min }$ is approximated by $\tilde{\mathbf{x}}_{\min }=\arg \min _{\mathbf{x}^{(j)}} f\left(\mathbf{x}^{(j)}\right), i=1, \ldots, S$ in the general case. The distances $d^{(j)}=d_{\mathrm{E}}\left(\mathbf{x}_{\min }, \mathbf{x}^{(j)}\right)$ (or $d^{(j)}=d_{\mathrm{E}}\left(\hat{\mathbf{x}}_{\min }, \mathbf{x}^{(j)}\right)$ ) and the corresponding fitness values $f^{(j)}$ provide a means to infer knowledge about the global topology of the landscape. A general low-dimensional landscape visualization can be achieved using fitness-distance scatter plots of the available samples. The structure of low-fitness landscape regions can be analyzed by sorting the $f^{(j)}$ and plotting only the $X \%$ best individuals. In addition to fitness-distance scatter plots, it may be informative to plot the histograms of the distances of the samples that have the $X \%$ best $f^{(j)}$ values. If the black-box problem has a clear double-funnel landscape topology, the resulting distance 
distributions are bi-modal. A single, scalar quantity that summarizes the fitness-distance data is the fitness-distance correlation coefficient $r_{\mathrm{FD}}$, defined as:

$$
r_{\mathrm{FD}}=\frac{c_{\mathrm{FD}}}{s_{\mathrm{F}} s_{\mathrm{D}}} \quad \text { with } \quad c_{\mathrm{FD}}=\frac{1}{S} \sum_{j=1}^{S}\left(f^{(j)}-\bar{f}\right)\left(d^{(j)}-\bar{d}\right)
$$

and $\bar{f}, \bar{d}, s_{\mathrm{F}}$, and $s_{\mathrm{D}}$ the means and standard deviations of the fitness and distance samples, respectively. The coefficient $r_{\mathrm{FD}}$ is expected to be near 1 for globally convex, single-funnel topologies and around 0 for needle-in-a-haystack problems and problems without any global structure. A negative value of $r_{\mathrm{FD}}$ indicates a "deceiving" landscape, i.e., a landscape on which a sampler or optimizer perceives larger objective function values closer to the minimum than farther away. It is obvious that the accuracy of $r_{\mathrm{FD}}$ is limited by sample size. If the landscape exhibits fine structures below the sampling limit, they cannot be detected. We thus do not consider $r_{\mathrm{FD}}$ as a "predictive measure of problem difficulty", as has been proposed for GA's and genetic programming [12]. In fact, it has been proven for certain problem classes that computing a general predictor of problem difficulty is as hard as solving the problem itself [17]. We rather emphasize that $r_{\mathrm{FD}}$ may represent one out of several potentially informative features based on which black-box landscapes can be classified.

\section{Characterization of the CEC 2005 Benchmark Test Suite}

We analyze the relationship between fitness and distance to the global minimum for all 25 functions $f_{i}$ of the CEC 2005 benchmark test suite [18], except for $f_{7}$ and $f_{25}$. The latter problems are unconstrained and would require the definition of an applicationspecific region of interest. Apart from the missing constraints, however, $f_{25}$ is identical to $f_{24}$. We choose the CEC 2005 benchmark because (i) the global topology of most functions is known a priori, and (ii) it enables relating $r_{\mathrm{FD}}$ to the performance of a large number of algorithms tested on this benchmark [19]6]. In order to enable this direct comparison, we use the standard settings that are also used for benchmarking optimization algorithms. This entails considering all problems in $n=10,30,50$ dimensions with the standard restriction on the function evaluations (FES) budget (MAX_FES $=10^{4} n$ ) and 25 repetitions per run. We present fitness-distance plots and distance distributions for selected functions. We then provide a comparative analysis of the FDC coefficients.

\subsection{Fitness-Distance Plots and Distance Distributions}

We first present scatter plots of the fitness and distance data for all CEC functions in Fig. 2. We focus on the 10-dimensional case. The scatter plots look similar also in higher dimensions (data not shown). Visual inspection of the plots in Fig. 2 2 reveals a rich diversity of patterns. Function $f_{1}$ can be clearly identified as the sphere function. Fitness-distance plots of $f_{6}, f_{9}$, and $f_{10}$ show strong positive correlations. For functions $f_{8}, f_{11}$, and $f_{14}$ the spherical scatter patterns suggest a complete absence of correlation.

Functions $f_{2}-f_{5}, f_{12}$, and $f_{13}$ show a similar pattern, suggesting weak correlations between fitness and distance in all these cases. The scatter plots for $f_{18}-f_{24}$ reveal that many samples far away from the minimum have considerably lower objective function 

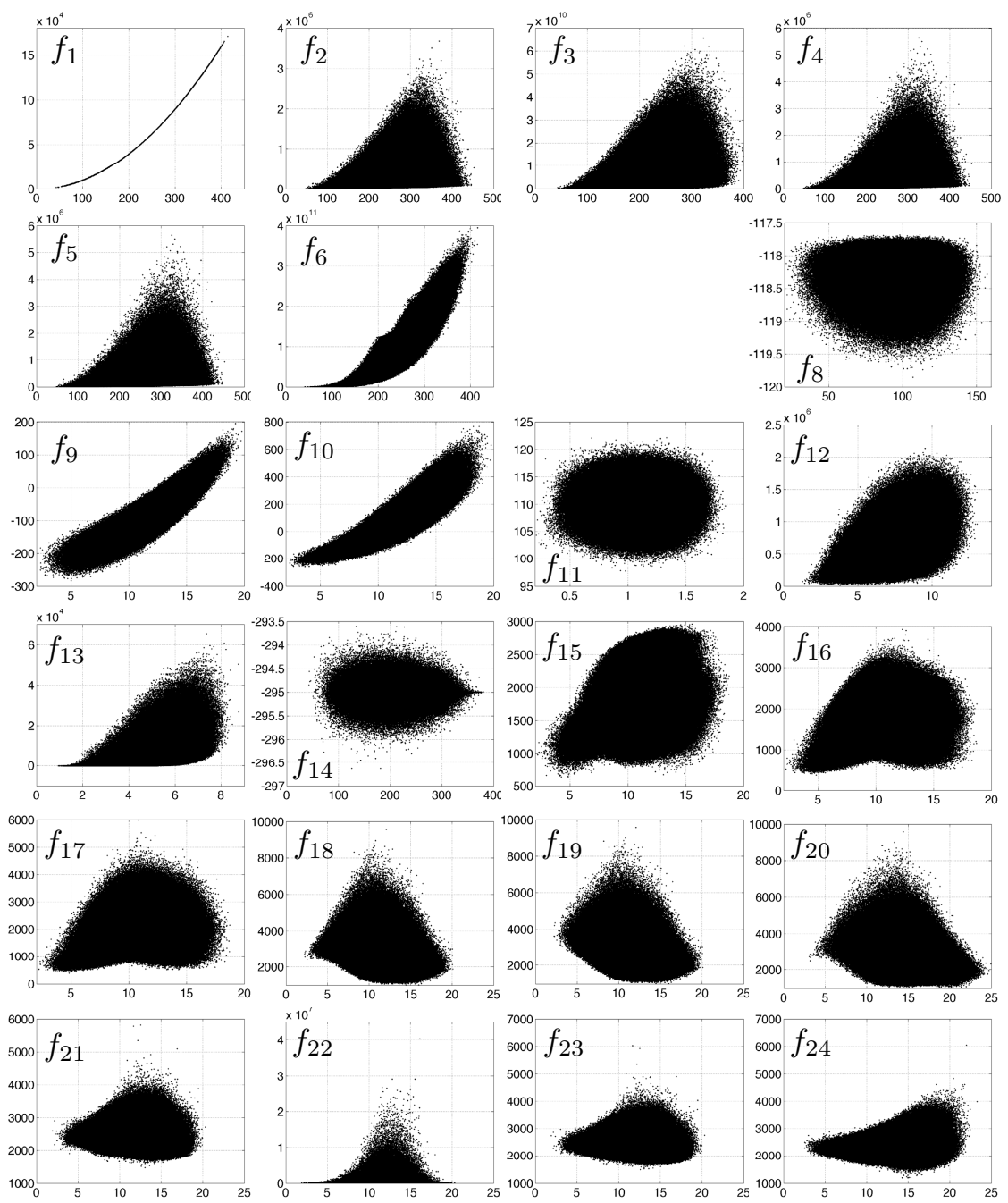

Fig. 2. Fitness $f_{i}$ versus distance to the global minimum $d_{\mathrm{E}}\left(\mathbf{x}_{\min }, \mathbf{x}\right)$ for all CEC functions except $f_{7}$ and $f_{25}$ in $n=10$ dimensions. The FES budget is limited to $10^{4} n$. The pooled samples from all 25 repetitions are shown.

values than samples close to the global minimum, characterizing these problems as "deceiving". An unique scatter plot pattern is observed for the triplet $f_{15}-f_{17}$. For samples with low objective function values, two distinct distance regimes are visible, which may suggest a double-funnel topology of the landscape.

We exemplify the use of scatter plots and distance histograms of samples with low objective function values for the pair of functions $f_{9}$ and $f_{15}$ that have the same landscape domain $[-5,5]^{10}$. The function $f_{9}$ is a shifted Rastrigin function with a globally convex topology. The function $f_{15}$ is a composite function, designed to have two distant 
regions of low objective function values [18]. Two-dimensional graphs of these functions are depicted in the upper panels of Fig. 3, For all 25 repetitions we identify the samples with the $1 \%$ best fitness values on $f_{9}$ and $f_{15}$ and present the corresponding fitness-distances scatter plot in the lower panels of Fig. 3 . While the samples on $f_{9}$ cluster around an average distance of $d_{\mathrm{E}}\left(\mathbf{x}_{\min }, \mathbf{x}\right) \approx 6$ in a fitness interval [-280,-200], the samples on $f_{15}$ show two distinct modes at $d_{\mathrm{E}}\left(\mathrm{x}_{\min }, \mathbf{x}\right) \approx 6$ and $d_{\mathrm{E}}\left(\mathbf{x}_{\min }, \mathbf{x}\right) \approx 11$ with fitness values ranging from $[800,1100]$. The corresponding distance histograms (Fig. (4) are unimodal for $f_{9}$ and bi-modal for $f_{15}$.
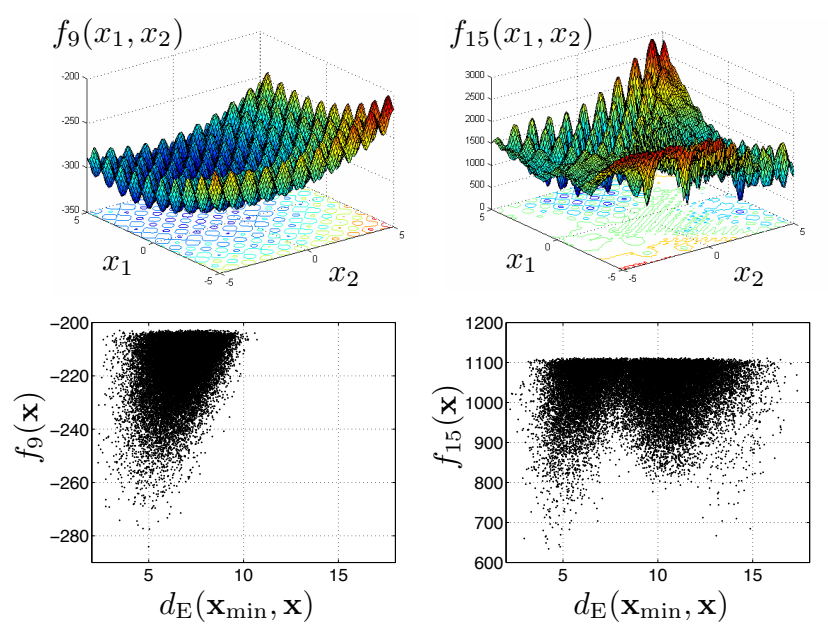

Fig. 3. Top row: 2D plots of the fitness landscapes of the CEC functions $f_{9}$ (upper-left panel) and $f_{15}$ (upper-right panel). The function $f_{9}$ is a shifted Rastrigin function, the function $f_{15}$ is a composite function with a double-funnel topology. Bottom row: Fitness-distance plots of the best $1 \%$ of the samples for $f_{9}$ (lower-left panel) and $f_{15}$ (lower-right panel) in $n=10$ dimensions. The pooled samples from all 25 repetitions are shown.
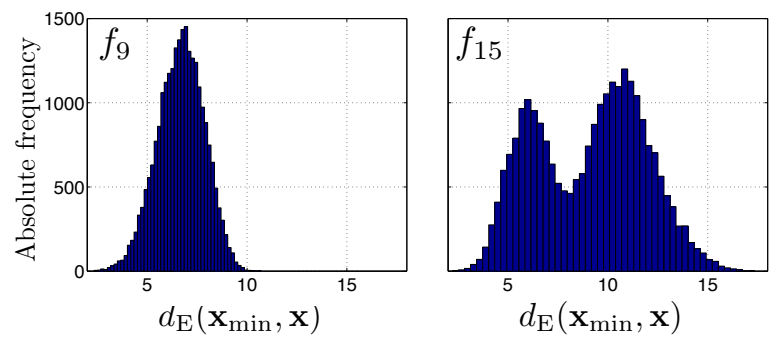

Fig. 4. Distribution of distances to the global minimum using the best $1 \%$ of the samples on $f_{9}$ and $f_{15}$ in $n=10$ dimensions, respectively. The pooled samples from all 25 repetitions are shown. The distance distribution on $f_{9}$ is unimodal, whereas the distance distribution on $f_{15}$ is bi-modal. 


\subsection{The Fitness-Distance Correlation Coefficients}

We summarize the calculated FDC coefficients $r_{\mathrm{FD}}$ in Fig. 5 The data suggest a rough classification of the functions into three classes: (i) highly correlated $r_{\mathrm{FD}}>0.75$, (ii) weakly correlated $0.75>r_{\mathrm{FD}}>0.15$, and (iii) uncorrelated or anti-correlated $r_{\mathrm{FD}}<$ 0.15 across all dimensions. Only functions $f_{18}, f_{19}$, and $f_{24}$ change class in higher dimensions.

The functions $f_{1}, f_{6}$, and $f_{9} / f_{10}$ belong to the first class. This indicates a global single-funnel topology. The shifted sphere function $f_{1}$ is expected to follow this classification. The shifted/rotated Rosenbrock function $f_{6}$, however, is multimodal. Nonetheless, the $r_{\mathrm{FD}}$ suggests that this multi-modality only appears at small length scales. The Rastrigin pair $f_{9} / f_{10}$ is also expected to yield large $r_{\mathrm{FD}}$ values because of its globally spherical structure. Comparing the two functions of this pair also reveals that the rotation in $f_{10}$ does not significantly change the estimated $r_{\mathrm{FD}}$ value.

In all dimensions, the set of weakly correlated functions comprises $f_{2}-f_{5}, f_{12}-f_{13}$, and $f_{15}-f_{17}$. While $f_{2}-f_{5}$ are unimodal functions, all others are highly multimodal with little or no globally convex structure. The $r_{\mathrm{FD}}$ values cannot discriminate these functions. The similar $r_{\mathrm{FD}}$ values for the function pairs $f_{2} / f_{4}$ and $f_{16} / f_{17}$ indicate that the measure is robust against noise. Among all hybrid functions $\left(f_{14}-f_{25}\right)$, the $r_{\mathrm{FD}}$ suggest that the triplet $f_{15}-f_{17}$ has the highest degree of global correlation.

The class of un-/anti-correlated functions contains $f_{8}, f_{11}, f_{14}$, and $f_{20}-f_{23}$ across all dimensions. For these functions, a low fitness-distance correlation is expected. For instance, $f_{8}$ is a needle-in-a-haystack problem and $f_{14}$ 's global minimum is surrounded by regions of alternating high and low objective function values whose amplitude decreases with increasing distance (see Fig. (2). The $r_{\mathrm{FD}}$ values for the pair $f_{18} / f_{19}$ change from anti-correlation in $n=10$ to weak correlation in $n=30,50$. This indicates that certain topological features that have been picked up by the measure in $n=10$ dimensions cannot be detected any more in higher dimensions, probably due to sampling limitations.
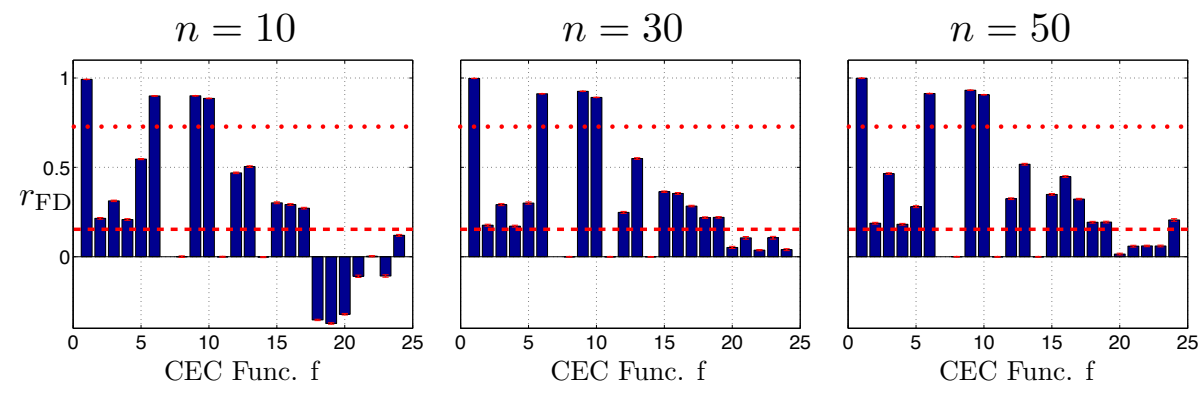

Fig. 5. Estimated FDC coefficients (mean and std) for all CEC functions except $f_{7}$ and $f_{25}$ in $n=10,30,50$ dimensions. The FES budget is limited to $10^{4} n$. The dotted line and the dashed line represent the classification thresholds (see main text). 


\section{Conclusions and Future Work}

Characterizing topographical features of continuous black-box landscapes from zerothorder information only is a formidable challenge that has largely been neglected in the black-box optimization community. In theoretical biology and discrete combinatorial optimization, the concept of fitness-distance correlations (FDC) has been studied over two decades. We adopted this framework for continuous black-box landscapes and introduced the corresponding quantities and measures. We have empirically analyzed the accuracy and robustness (against noise, problem rotation, and dimensionality) of fitness-distance analysis on the CEC 2005 test functions with known global topologies. The results have shown that the CEC 2005 benchmark functions cover a wide spectrum of FDC coefficient values. This contradicts recent results of Vanneschi and co-workers [20] who claim that the CEC 2005 test functions only have FDC coefficients close to one or zero.

Our findings on the benchmark suite have also shown that fitness-distance correlation can discriminate between functions with a global single-funnel topology, such as the Rastrigin function $\left(f_{9}\right)$, and highly unstructured problems, such as the needle-ina-haystack problem $\left(f_{8}\right)$. Moreover, we found FDC to be robust against noise $\left(f_{2}, f_{4}\right)$, problem rotation $\left(f_{9} / f_{10}\right)$, and dimensionality, provided a sufficiently large ensemble of samples is considered. The present benchmark, however, also illustrated the limitations of the approach: FDC was not able to distinguish anisotropic quadratic functions $\left(f_{2} / f_{3}\right)$ from multimodal $\left(f_{12}-f_{13}\right)$ and multi-funnel $\left(f_{15}-f_{17}\right)$ problems, although the former are smooth and unimodal. These results, together with the known performance of search heuristics on these functions [19]6], also suggest that FDC coefficients alone are neither a meaningful criterion for problem design nor for measuring problem difficulty. This is again in stark contrast to the suggestions made by Vanneschi and coworkers [20]. We argue that fitness-distance analysis can only provide one out of several useful landscape descriptors that need to be combined in order to form discriminative "landscape fingerprints". We envision these fingerprints to be used in a future classification of black-box landscapes.

\section{References}

1. Wright, S.: The roles of mutation, inbreeding, crossbreeding, and selection in evolution. In: Proceedings of the Sixth International Congress on Genetics (1932)

2. Boese, K.D., Kahng, A.B., Muddu, S.: A new adaptive multi-start technique for combinatorial global optimizations. Operations Research Letters 16(2), 101-113 (1994)

3. Hu, T.C., Klee, V., Larman, D.: Optimization of globally convex functions. SIAM Journal on Control and Optimization 27(5), 1026-1047 (1989)

4. Hansen, N., Kern, S.: Evaluating the CMA Evolution Strategy on Multimodal Test Functions. In: Yao, X., Burke, E.K., Lozano, J.A., Smith, J., Merelo-Guervós, J.J., Bullinaria, J.A., Rowe, J.E., Tiňo, P., Kabán, A., Schwefel, H.-P. (eds.) PPSN VIII 2004. LNCS, vol. 3242, pp. 282-291. Springer, Heidelberg (2004)

5. Lunacek, M., Whitley, D.: The Dispersion Metric and the CMA Evolution Strategy. In: GECCO 2006: Proceedings of the 8th Annual Conference on Genetic and Evolutionary Computation, pp. 477-484. ACM Press, New York (2006) 
6. Müller, C.L., Baumgartner, B., Sbalzarini, I.F.: Particle Swarm CMA Evolution Strategy for the optimization of multi-funnel landscapes. In: Proc. of IEEE Congress on Evolutionary Computation (CEC 2009), pp. 2685-2692 (May 2009)

7. Sakuma, J., Kobayashi, S.: Extrapolation-directed crossover for real-coded ga: overcoming deceptive phenomena by extrapolative search. In: Proceedings of the 2001 Congress on Evolutionary Computation, vol. 1, pp. 655-662 (2001)

8. Lunacek, M., Whitley, D., Sutton, A.: The Impact of Global Structure on Search. In: Rudolph, G., Jansen, T., Lucas, S., Poloni, C., Beume, N. (eds.) PPSN X 2008. LNCS, vol. 5199, pp. 498-507. Springer, Heidelberg (2008)

9. Müller, C.L., Sbalzarini, I.F.: A Tunable Real-world Multi-funnel Benchmark Problem for Evolutionary Optimization - And Why Parallel Island Models Might Remedy the Failure of CMA-ES on It. In: Dourado, A., Rosa, A.C., Madani, K. (eds.) Proc. of the International Joint Conference on Computational Intelligence (IJCCI), October 5-7, pp. 248-253 (2009)

10. Kauffman, S.A.: The Origins of Order: Self-Organization and Selection in Evolution, 1st edn. Oxford University Press, USA (1993)

11. Kauffman, S.A., Weinberger, E.D.: The NK model of rugged fitness landscapes and its application to maturation of the immune response. Journal of Theoretical Biology 141(2), 211245 (1989)

12. Jones, T., Forrest, S.: Fitness distance correlation as a measure of problem difficulty for genetic algorithms. In: Proceedings of the 6th International Conference on Genetic Algorithms, pp. 184-192. Morgan Kaufmann Publishers Inc., San Francisco (1995)

13. Merz, P., Freisleben, B.: Memetic Algorithms and the Fitness Landscape of the Graph BiPartitioning Problem. In: Eiben, A.E., Bäck, T., Schoenauer, M., Schwefel, H.-P. (eds.) PPSN V 1998. LNCS, vol. 1498, p. 765. Springer, Heidelberg (1998)

14. Ikeda, K., Kobayashi, S.: GA Based on the UV-Structure Hypothesis and Its Application to JSP. In: PPSN VI: Proceedings of the 6th International Conference on Parallel Problem Solving from Nature, London, UK, pp. 273-282. Springer, Heidelberg (2000)

15. Merz, P.: Advanced fitness landscape analysis and the performance of memetic algorithms. Evol. Comput. 12(3), 303-325 (2004)

16. Wang, Y., Li, B.: Understand behavior and performance of real coded optimization algorithms via nk-linkage model. In: IEEE Congress on Evolutionary Computation, CEC 2008. IEEE World Congress on Computational Intelligence, pp. 801-808 (1-6, 2008)

17. He, J., Reeves, C., Witt, C., Yao, X.: A note on problem difficulty measures in black-box optimization: Classification, realizations and predictability. Evol. Comput. 15(4), 435-443 (2007)

18. Suganthan, P.N., Hansen, N., Liang, J.J., Deb, K., Chen, Y.P., Auger, A., Tiwari, S.: Problem Definitions and Evaluation Criteria for the CEC 2005 Special Session on Real-Parameter Optimization. Technical report, Nanyang Technological University, Singapore (May 2005)

19. Hansen, N.: Compilation of Results on the 2005 CEC Benchmark Function Set. Technical report, Computational Laboratory (CoLab), Institute of Computational Science, ETH Zurich (2006)

20. Vanneschi, L., Codecasa, D., Mauri, G.: An empirical comparison of parallel and distributed particle swarm optimization methods. In: Proceedings of the 12th Annual Conference on Genetic and Evolutionary Computation, GECCO 2010, pp. 15-22. ACM, New York (2010) 